Rev. Int. Contam. Ambie. 32 (3) 339-351, 2016

DOI: 10.20937/RICA.2016.32.03.08

\title{
AISLAMIENTO Y EVALUACIÓN DE LA ACTIVIDAD ENZIMÁTICA LIGNINOLÍTICA DE MACROMICETOS DEL ESTADO DE VERACRUZ, MÉXICO
}

\author{
Wilberth CHAN CUPUL ${ }^{1 *}$, Gabriela Patricia HEREDIA ABARCA ${ }^{2}$ y \\ Refugio RODRÍGUEZ VÁZQUEZ ${ }^{3}$
}

${ }^{1}$ Laboratorio de Fitopatología y Control Biológico, Facultad de Ciencias Biológicas y Agropecuarias, Universidad de Colima, kilómetro 40 Autopista Colima-Manzanillo, Tecomán, Colima, México, C.P. 28100

${ }^{2}$ Laboratorio de Micromicetos, Instituto de Ecología A. C. Carretera Antigua a Coatepec 351, El Haya, Xalapa, Veracruz, México, C.P. 91070

${ }^{3}$ Laboratorio de Xenobióticos, Centro de Investigación y de Estudios Avanzados, Instituto Politécnico Nacional. Avenida IPN 2508, San Pedro Zacatenco, Ciudad de México, México, C.P. 07360

*Autor para correspondencia:wchan@ucol.mx

(Recibido mayo 2015; aceptado noviembre 2015)

Palabras clave: biotecnología ambiental, lacasa, manganeso peroxidasa, micobiota nativa, fermentación líquida y sólida

\section{RESUMEN}

La diversidad fúngica del estado de Veracruz, México, es una importante fuente para la obtención de enzimas con valor biotecnológico. El objetivo de este estudio fue aislar y evaluar cualitativa (placas de agar) y cuantitativamente (en fermentación líquida y sólida) la actividad enzimática ligninolítica de macromicetos nativos. De un total de 70 aislamientos, 41 presentaron actividad lacasa en la evaluación cualitativa. Por destacar en el ensayo cualitativo se seleccionaron las cepas Trametes maxima SM9, Pycnoporus sanguineus ACT1 y Daedalea elegans PM7 para la evaluación cuantitativa de la actividad lacasa y manganeso peroxidasa (MnP) en los medios de cultivo líquido: Bose, Koroljova y Sivakumar, así como en los residuos agroindustriales: paja de trigo, bagazo de caña y aserrín de pino. En el medio Sivakumar se detectó la mayor actividad lacasa y MnP. Trametes maxima SM9 presentó los valores más altos para lacasa $(9121.8 \mathrm{U} / \mathrm{mg}$ de proteína) y MnP (477.9 U/mg de proteína), en segundo término estuvo P. sanguineus ACT1 (lacasa $=5422.2 \mathrm{U} / \mathrm{mg}$ de proteína y $\mathrm{MnP}=41.0 \mathrm{U} / \mathrm{mg}$ de proteína) y Daedalea elegans $\mathrm{PM} 7$ (lacasa $=1784.2 \mathrm{U} / \mathrm{mg}$ de proteína y $\mathrm{MnP}=$ $40.0 \mathrm{U} / \mathrm{mg}$ de proteína). En fermentación sólida P. sanguineus ACT1 obtuvo la mayor actividad lacasa en paja de trigo (1409.0 U/mg de peso seco) y bagazo de caña (1404.8 $\mathrm{U} / \mathrm{mg}$ de peso seco). Mientras que la actividad MnP fue mejor sobre bagazo de caña tanto para $P$. sanguineus ACT1 y T. maxima SM9 con 619.0 y $519.6 \mathrm{U} / \mathrm{mg}$ de peso seco, respectivamente. Las cepas Trametes maxima SM9 y P. sanguineus ACT1 son consideradas como buenas candidatas para la producción de lacasa y MnP.

Key words: environmental biotechnology, laccase, manganese peroxidase, native mycobiota, submerged and solid state fermentation 


\begin{abstract}
Fungal diversity in the state of Veracruz, Mexico, is a source of enzymes with biotechnological value. The objective of this study was to isolate and evaluate qualitatively (in Petri dishes) and quantitatively (in liquid and solid fermentation) the ligninolytic enzyme activities of native macromycetes. Seventy isolates were made, and 41 showed laccase activity in the qualitative assessment. The best strains from the qualitative assessment were Trametes maxima SM9, Pycnoporus sanguineus ACT1 and Daedalea elegans PM7. These strains were chosen to evaluate the laccase and manganese peroxidase (MnP) activities in Bose, Koroljova and Sivakumar liquid culture media and for agroindustrial waste in wheat straw, sugar cane bagasse and pine sawdust. The highest laccase and MnP activities were obtained in Sivakumar culture medium. Trametes maxima showed the highest values for laccase (9121.8 U/ $\mathrm{mg}$ of protein $)$ and $\mathrm{MnP}(477.9 \mathrm{U} / \mathrm{mg}$ of protein $)$ activities, followed by $P$. sanguineus ACT1 (laccase $=5422.2 \mathrm{U} / \mathrm{mg}$ of protein and $\mathrm{MnP}=41.0 \mathrm{U} / \mathrm{mg}$ of protein) and $D$. elegans $\mathrm{PM} 7$ (laccase $=1784.2 \mathrm{U} / \mathrm{mg}$ of protein and $\mathrm{MnP}=40.0 \mathrm{U} / \mathrm{mg}$ of protein). In regards to the solid state fermentation, $P$. sanguineus $\mathrm{ACT} 1$ produced the highest laccase activity on wheat straw (1409.0 U/mg dry weight) and sugar cane bagasse (1404.8 U/mg dry weigh). Meanwhile, MnP activities were higher in sugar cane bagasse for P. sanguineus ACT1 and T. maxima SM9 with 619.0 and $519.6 \mathrm{U} / \mathrm{mg}$ dry weight, respectively. Trametes maxima SM9 and P. sanguineus ACT1 are considered good candidates for laccase and MnP production.
\end{abstract}

\section{INTRODUCCIÓN}

Los macromicetos lignícolas en su mayoría pertenecen al grupo de los basidiomicetos y son los organismos más eficientes en la degradación de la lignina, polímero natural de estructura compleja (Ruiz-Dueñas et al. 2014). Únicamente los hongos ligninolíticos y especialmente, los macromicetos lignícolas son capaces de mineralizar la lignina en conjunto con los otros componentes de la madera (Harris-Valle et al. 2014). En la degradación de estos compuestos están involucradas algunas enzimas fúngicas extracelulares tales como la lacasa (EC 1.10.3.2), manganeso peroxidasa (MnP, EC 1.11.1.14) y lignina peroxidasa (LiP, EC 1.11.1.13), las cuales poseen múltiples aplicaciones biotecnológicas en la industria papelera, textil, alimentaria, farmacéutica y ambiental (Osma et al. 2014).

Estudios recientes mencionan que la prospección enzimática en la micobiota nativa es una herramienta biotecnológica para enfrentar problemas ambientales como la contaminación del agua (Hernández-Luna et al. 2008, Tapia-Tussell et al. 2011 y Cruz-Ramírez et al. 2012). Se ha demostrado que la micobiota nativa posee la habilidad para decolorar agua contaminada con azul ácido 74 , verde reactivo 19, rojo reactivo 195 (Tapia-Tussell et al. 2011), antraquinona, cristal violeta y colorantes tipo azo (Hernández-Luna et al. 2008). Además, se ha comprobado que las enzimas ligninolíticas poseen la habilidad para transformar o degradar plaguicidas de diferente grupo estructural tales como las triazinas (Chan-Cupul et al. 2014), organoclorados (MarcoUrrea y Reddy 2012), organofosforados (Huifang et al. 2013), entre otros. Por lo tanto, el número de investigaciones dirigidas hacia la búsqueda y estudio de nuevas especies y cepas de macrohongos lignícolas con alta capacidad para producir enzimas ligninolíticas se ha incrementado considerablemente en las últimas décadas (Fernández-Fernández et al. 2013).

En México, la diversidad fúngica se calcula entre las 140000 y 200000 especies, de las cuales menos del $10 \%$ han sido estudiadas (Guzmán 1995). En cuanto a macromicetos se han descrito aproximadamente 4000 especies (Guzmán 1998). Respecto a la magnitud de la riqueza de las especies fúngicas por entidad federativa aún se está lejos de contar con un conocimiento representativo. Sin embargo, el estado de Veracruz posee el mayor número de especies descritas, habiéndose registrado 1517 (Guzmán et al. 2003, Aguirre-Acosta et al. 2014). Pese a que México es uno de los países con mayor diversidad fúngica, se han realizados pocos estudios de prospección enzimática en macrohongos lignícolas para encontrar especies productoras de enzimas de importancia biotecnológica. Entre los estudios realizados destacan los trabajos de Hernández-Luna et al. (2008), TapiaTussell et al. (2011), Cruz-Ramírez et al. (2012) y Chan-Cupul (2014) desarrollados con especímenes 
colectados en los estados de Nuevo León, Yucatán, Hidalgo y Veracruz, respectivamente.

La prospección de la riqueza fúngica nativa en la búsqueda de cepas con potencial para la producción de enzimas de importancia biotecnológica, es una actividad prioritaria ante la constante pérdida de la biodiversidad de los ecosistemas locales. Por lo tanto, el presente estudio tuvo como objetivos aislar y seleccionar macromicetos nativos del estado de Veracruz con potencial para la producción de enzimas ligninolíticas. Asimismo, para las cepas con mayor potencial enzimático, se pretendió evaluar su actividad lacasa y manganeso peroxidasa (MnP) en tres medios de cultivo líquido y valorar así la producción de ambas enzimas bajo condiciones de fermentación sólida (paja de trigo, bagazo de caña y aserrín de pino).

\section{MATERIALES Y MÉTODOS}

\section{Colecta, aislamiento e identificación de macromi- cetos lignícolas}

Los carpóforos fueron colectados de diversos sitios en nueve municipios del estado de Veracruz, México en los meses de septiembre de 2011 a abril de 2012 (Cuadro I). El aislamiento del micelio se realizó a través de la desinfección de fragmentos
(0.5-1.0 $\left.\mathrm{cm}^{2}\right)$ de los carpóforos con hipoclorito de sodio (5\%), alcohol etílico (70 \%) y agua destilada. Los fragmentos desinfectados fueron depositados sobre placas con agar papa dextrosa (APD) suplementado con cloranfenicol (Lab. Sophia, México) y benomyl (Biesterfeld Co. USA) a una concentración de 3 ppm, para evitar el crecimiento de bacterias y hongos microscópicos (Chaparro et al. 2009). Las placas inoculadas se incubaron a $25{ }^{\circ} \mathrm{C}$ durante 8 días (d), el micelio emergente se transfirió a cajas de Petri con APD para la obtención de cultivos puros. Las cepas aisladas se conservaron a $4{ }^{\circ} \mathrm{C}$ en APD. La identificación de los carpóforos (Fig. 1) que dieron origen al micelio fueron identificados con base en sus características morfológicas por medio de claves taxonómicas especializadas (Gilbertson y Ryvarden 1987, Ryvarden 1991, Núñez y Ryvarden 2001, Guzmán 2008).

\section{Determinación cualitativa de la actividad enzimá- tica ligninolítica}

Para seleccionar las cepas enzimáticamente activas, se realizaron ensayos de coloración del ácido 2,2'-azino-bis[3-etilbenzotiazolin-6-sulfónico] (ABTS) en el medio de cultivo descrito por Rubilar-Araneda (2007), el cual contiene (g/L): glucosa (10), $\mathrm{KH}_{2} \mathrm{PO}_{4}$ (2), $\mathrm{MgSO}_{4} \cdot 7 \mathrm{H}_{2} \mathrm{O}(0.5), \mathrm{CaCl}_{2} \cdot 2 \mathrm{H}_{2} \mathrm{O}(0.1)$, tartrato de amonio (0.2), ABTS (0.2) y agar (22) a un pH de 5.5.

CUADRO I. MUNICIPIOS Y LOCALIDADES INCLUIDOS EN LA COLECTA DE MACROMICETOS LIGNÍCOLAS EN EL ESTADO DE VERACRUZ, MÉXICO

\begin{tabular}{|c|c|c|c|}
\hline Municipio & Localidades & Clave & Ubicación \\
\hline \multirow{2}{*}{ Actopan } & Actopan & $\mathrm{ACT}$ & $19^{\circ} 30 " 21.16^{\prime \prime} \mathrm{N}, 96^{\circ} 36^{\prime \prime} 31.23 " \mathrm{O}$ \\
\hline & Centro de Investigaciones Costeras la Mancha & CLM & $19^{\circ} 35^{\prime \prime} 36.96^{\prime \prime} \mathrm{N}, 96^{\circ} 22^{\prime} 51.79^{\prime \prime} \mathrm{O}$ \\
\hline Acajete & Mesa de hierba & $\mathrm{MH}$ & $19^{\circ} 32^{\prime \prime 43.11 ” N, ~} 96^{\circ} 59 " 43.11^{\prime \prime} \mathrm{O}$ \\
\hline \multirow{3}{*}{ Coatepec } & Coatepec & $\mathrm{COA}$ & $19^{\circ} 28^{\prime \prime} 15.71 ” \mathrm{~N}, 96^{\circ} 577^{\prime \prime} 31.12 " \mathrm{O}$ \\
\hline & Paso de la loma & PM & $19^{\circ} 30 " 18.99 ” \mathrm{~N}, 96^{\circ} 57^{\prime \prime 19.62 " ~ O ~}$ \\
\hline & Palo blanco & PB & $19^{\circ} 32 " 15.71^{\prime \prime N}, 97^{\circ} 00 " 23.38^{\prime \prime} \mathrm{O}$ \\
\hline Huatusco & Las cañadas & CÑDA & $19^{\circ} 09 “ 52.86 ” \mathrm{~N}, 96^{\circ} 58^{\prime \prime} 48.84 ” \mathrm{O}$ \\
\hline \multirow{3}{*}{ San Andrés Tuxtlas } & Adolfo Ruiz Cortines & SAT & $18^{\circ} 32 “ 58.90 " \mathrm{~N}, 95^{\circ} 09^{\prime \prime} 37.75^{\prime \prime} \mathrm{O}$ \\
\hline & Santa Marta & SM & $18^{\circ} 19^{\prime \prime} 10.83^{\prime \prime N}, 94^{\circ} 49^{\prime \prime} 55.69^{\prime \prime} \mathrm{O}$ \\
\hline & Playa escondida & $\mathrm{PE}$ & $18^{\circ} 35^{\prime \prime} 32.68^{\prime \prime} \mathrm{N}, 95^{\circ} 03^{\prime \prime} 01.65^{\prime \prime} \mathrm{O}$ \\
\hline Tlalnelhuayocan & Vega del Pixquiac & $\mathrm{VP}$ & $19^{\circ} 32 " 21.23 ” \mathrm{~N}, 97^{\circ} 00 " 47.29^{\prime \prime} \mathrm{O}$ \\
\hline Veracruz & Humedal Veracruzano & $\mathrm{HV}$ & $19^{\circ} 08^{\prime \prime} 35.70^{\prime \prime} \mathrm{N}, 96^{\circ} 09^{\prime} 05.57^{\prime \prime} \mathrm{O}$ \\
\hline \multirow{3}{*}{ Xalapa } & Santuario de bosque de niebla & SBN & $19^{\circ} 30 “ 48.53 ” \mathrm{~N}, 96^{\circ} 56 “ 33.10^{\prime \prime} \mathrm{O}$ \\
\hline & Jardín Botánico Francisco Javier Clavijero & JB & $19^{\circ} 30 " 44.40^{\prime \prime} \mathrm{N}, 96^{\circ} 56^{\prime \prime} 32.55^{\prime \prime} \mathrm{O}$ \\
\hline & Parque vivero & PV & $19^{\circ} 30^{\circ} 51.07^{\prime \prime} \mathrm{N}, 96^{\circ} 56^{\prime \prime} 38.53 ” \mathrm{O}$ \\
\hline Xico & Cascadas de Texolo & TXL & $19^{\circ} 24^{\prime \prime} 06.55^{\prime \prime} \mathrm{N}, 96^{\circ} 59^{\prime \prime} 38.76^{\prime \prime} \mathrm{O}$ \\
\hline
\end{tabular}




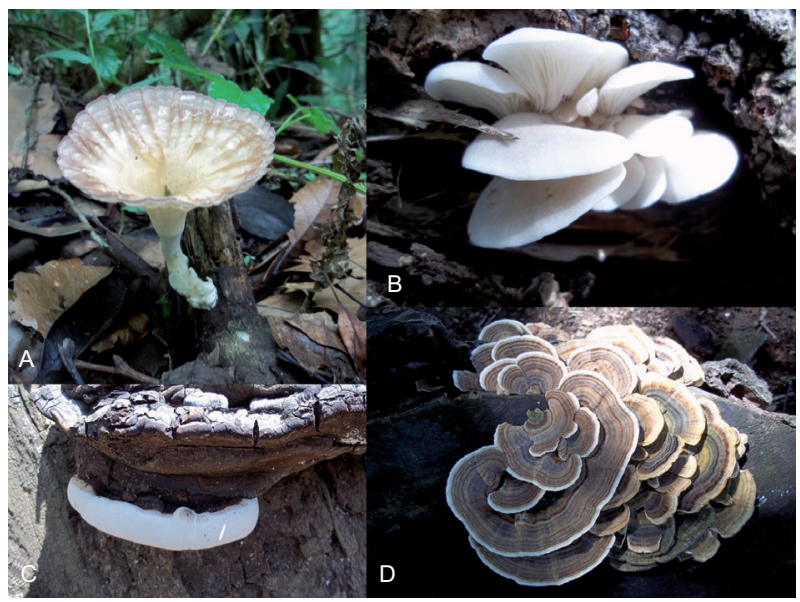

Fig. 1. Carpóforos colectados en campo. A) Cymatoderma elegans SBN4, B) Pleurotus sp. SBN8, C) Ganoderma sp. PB1 y D) Trametes sp. SBN2

El medio de cultivo se esterilizó en autoclave a $120^{\circ} \mathrm{C}$ durante $15 \mathrm{~min}$, el ABTS se añadió al enfriarse dicho medio de cultivo a $40-45^{\circ} \mathrm{C}$ y se distribuyó en cajas de Petri de $90 \mathrm{~mm}$ de $\varnothing$. Posteriormente, se extrajo un disco de los hongos aislados en APD y se colocó en el centro de la caja Petri, las placas se incubaron $25{ }^{\circ} \mathrm{C}$ durante $8 \mathrm{~d}$. La actividad enzimática (lacasa) se consideró positiva ante la presencia de un halo de oxidación de color verde-oscuro a verde-esmeralda (Rubilar-Araneda 2007). Cada 24 h se cuantificó el diámetro de la colonia del hongo y el diámetro del halo de oxidación del ABTS mediante una escuadra milimétrica. Con ambos valores, se calculó el índice de potencia (IP) dividiendo el diámetro del halo de oxidación entre el diámetro de crecimiento del micelio, para cada cepa se inocularon cuatro placas.

\section{Actividad enzimática ligninolítica en fermenta- ción líquida}

Se seleccionaron tres cepas aisladas con el mayor IP para cuantificar la producción de lacasa y manganeso peroxidasa $(\mathrm{MnP})$ en tres diferentes medios de cultivo líquido, los cuales fueron seleccionados por ser reportados en literatura científica como medios para la producción de enzimas ligninolíticas (Cuadro II). Los medios de cultivo se esterilizaron y se ajustaron a un $\mathrm{pH}$ de 4.5. Para la inoculación se depositaron cuatro discos de micelio agar $(5 \mathrm{~mm}$ de $\varnothing)$ en un matraz Erlenmeyer de $250 \mathrm{~mL}$ de capacidad con $120 \mathrm{~mL}$ del medio de cultivo. Los matraces inoculados fueron incubados en agitación orbital a $120 \mathrm{rpm}, 25 \pm 1{ }^{\circ} \mathrm{C}$ y en ausencia de luz durante 12 d. Diariamente se extrajeron asépticamente alícuotas de $3 \mathrm{~mL}$ del extracto de cultivo para la cuantificación
CUADRO II. COMPOSICIÓN NUTRIMENTAL (g/L) DE LOS MEDIOS DE CULTIVO LÍQUIDO EVALUADOS PARA LA PRODUCCIÓN DE ENZIMAS LIGNINOLÍTICAS.

\begin{tabular}{lccc}
\hline Componente & $\begin{array}{c}\text { Bose } \\
\text { et al. }(2007)\end{array}$ & $\begin{array}{c}\text { Koroljova } \\
\text { et al. }(1998)\end{array}$ & $\begin{array}{c}\text { Sivakumar } \\
\text { et al. }(2010)\end{array}$ \\
\hline $\begin{array}{l}\text { Glucosa } \\
\text { Extracto de }\end{array}$ & 20 & 10.0 & 20.0 \\
levadura & 5.0 & - & \\
Peptona & 2.0 & 3.0 & -5 \\
$\mathrm{KH}_{2} \mathrm{PO}_{4}$ & 1.5 & 0.6 & - \\
$\mathrm{K}_{2} \mathrm{HPO}_{4}$ & - & 0.4 & - \\
$\left(\mathrm{NH}_{4}\right)_{2} \mathrm{SO}_{4}$ & 1.0 & - & 0.05 \\
$\mathrm{MgSO}_{4}$ & 0.5 & 0.5 & 0.5 \\
$\mathrm{CaCl}_{2}$ & 0.37 & - & 0.01 \\
$\mathrm{FeSO}_{4}$ & - & 0.0005 & 0.01 \\
$\mathrm{MnSO}_{4}$ & - & 0.05 & 0.001 \\
$\mathrm{ZnSO}_{4}$ & 0.3 & 0.001 & 0.001 \\
$\mathrm{CuSO}_{4}$ & - & - & 0.002 \\
\hline
\end{tabular}

de la actividad de las enzimas lacasa y MnP, además del contenido de proteína. Previo a la cuantificación enzimática, el extracto de cultivo fue centrifugado a 7500 rpm durante $10 \mathrm{~min}$ para liberar el sobrenadante de los restos celulares. Para cada cepa se emplearon tres réplicas por medio de cultivo.

\section{Actividad enzimática ligninolítica en fermenta- ción sólida}

Para conocer el potencial de las tres cepas con mayor IP para la producción de enzimas en sustratos orgánicos de bajo costo respecto a los cultivos en medios líquidos, se evaluó la producción de lacasa y $\mathrm{MnP}$ en tres sustratos agroindustriales disponibles para el estado de Veracruz, los cuales fueron: paja de trigo, bagazo de caña y aserrín de pino. Para ello, se preparó el inóculo que consistió en la reproducción masiva del micelio en semillas de sorgo (Sorghum vulgare Pers.). Las semillas se tamizaron para eliminar residuos y se hidrataron en agua por $24 \mathrm{~h}$ hasta obtener aproximadamente un $75 \%$ de humedad. En bolsas de polipapel se depositaron $120 \mathrm{~g}$ de semillas de sorgo hidratadas y se esterilizaron a $125^{\circ} \mathrm{C}$ por 15 min. Una vez que las semillas se enfriaron a temperatura ambiente, se abrieron las bolsas asépticamente y se les depositaron 10 discos de micelio agar $(5 \mathrm{~mm}$ de Ø) de las cepas a estudiar. Las bolsas se incubaron a $25 \pm 1{ }^{\circ} \mathrm{C}$ por $8 \mathrm{~d}$. La bolsa con semillas de sorgo colonizadas se consideró como inóculo (Mata et al. 2011).

Para el crecimiento de los hongos en los residuos agroindustriales se homogeneizó cada sustrato a un tamaño de partícula de $0.5 \mathrm{~cm}$ a través del trozado y 
tamizado. Se pesaron cinco g de cada residuo agroindustrial, llevándolos al $100 \%$ de su capacidad de retención de humedad y se depositaron en matraces Erlenmeyer de $250 \mathrm{~mL}$ de capacidad. Los matraces se esterilizaron a $125^{\circ} \mathrm{C}$ durante $20 \mathrm{~min}$, posteriormente se les adicionó el inóculo preparado previamente al $5 \%(0.5 \mathrm{~g})$. Los matraces inoculados fueron incubados estáticamente a $25 \pm 1{ }^{\circ} \mathrm{C}$ en ausencia de luz durante $30 \mathrm{~d}$. Se emplearon tres réplicas para cada residuo agroindustrial por cepa evaluada. Cada $72 \mathrm{~h}$ se colectó asépticamente $0.5 \mathrm{~g}$ de residuo agroindustrial para la cuantificación de la actividad lacasa, MnP y contenido de proteína. Para ello, se realizó un extracto enzimático que consistió en depositar $0.5 \mathrm{~g}$ de residuo agroindustrial colonizado en un tubo falcón con $5 \mathrm{~mL}$ de amortiguador acetato de sodio $\mathrm{pH} 4$.5. Los tubos se agitaron horizontalmente durante $20 \mathrm{~min}$, posteriormente, fueron centrifugados a $5000 \mathrm{rpm}$ durante 10 min para sedimentar las partículas sólidas. El sobrenadante se empleó para la cuantificación enzimática.

\section{Evaluación de la actividad enzimática}

Las actividades lacasa, MnP y contenido de proteína se cuantificaron espectrofotométricamente. Para la actividad lacasa fue por la oxidación del ABTS (Sunil et al. 2011) a temperatura ambiente $\left(27-25^{\circ} \mathrm{C}\right)$ enunareacción que contiene $5.0 \mathrm{mMdeABTS}(100 \mu \mathrm{L})$, $100 \mathrm{mM}$ de amortiguador acetato de sodio $(800 \mu \mathrm{L}$, $\mathrm{pH} 4.5)$ y extracto enzimático $(100 \mu \mathrm{L})$; el cambio en la absorbancia se midió durante 5 min a 420 $\mathrm{nm}(\varepsilon=36000 \mathrm{mM} / \mathrm{cm})$. Una unidad de actividad lacasa (U) se definió como la cantidad de enzima requerida para oxidar $1 \mu \mathrm{mol}$ de $\mathrm{ABTS} / \mathrm{mL} / \mathrm{min}$ y se expresó como actividad específica (U/mg de proteína o U/mg de peso seco) y volumétrica (U/L) tanto en la fermentación líquida como en la sólida.

La actividad MnP se determinó por el método de Glenn y Gold (1983). La mezcla de reacción contenía lactato de sodio $25 \mathrm{mM}(50 \mu \mathrm{L}), \mathrm{MnSO}_{4} 2 \mathrm{mM}(50 \mu \mathrm{L})$, albúmina de huevo al $0.1 \%(50 \mu \mathrm{L})$, rojo fenol al $2 \%(50 \mu \mathrm{L})$, amorgituador succinato-lactato ( $\mathrm{pH} 4.5)$ $20 \mathrm{mM}(50 \mu \mathrm{L})$ y extracto enzimático $(700 \mu \mathrm{L})$. La reacción se inició al adicionar $50 \mu \mathrm{L}$ de $\mathrm{H}_{2} \mathrm{O}_{2}(2 \mathrm{mM})$ y se detuvo a los cinco min con $50 \mu \mathrm{L}$ de $\mathrm{NaOH}(2 \mathrm{~N})$. $\mathrm{La}$ absorbancia fue leída contra un testigo $\sin \mathrm{MnSO}_{4}$ en la reacción. El coeficiente de extinción molar del rojo fenol bajo las condiciones de reacción fue de 4460 $\mathrm{mM} / \mathrm{cm}$. Una actividad MnP (U) se definió como la cantidad de enzima requerida para transformar $1 \mu \mathrm{mol}$ de rojo fenol $/ \mathrm{mL} / \mathrm{min}$ y se expresó como actividad específica (U/mg de proteína o U/mg de peso seco) y volumétrica $(\mathrm{U} / \mathrm{L})$ tanto en la fermentación líquida como en la sólida.
El contenido de proteína total en fermentación líquida se cuantificó a través del método de Bradford en el que se emplea una curva patrón con valores conocidos $(0,0.0062,0.0125,0.025,0.05,0.1$ y $0.2 \mathrm{mg} /$ $\mathrm{mL}$ ) y albúmina de suero bovino como estándar. La curva patrón fue de $\mathrm{Y}=0.1615 \mathrm{C}-0.0125(\mathrm{Y}=\mathrm{ab}-$ sorbancia, $\mathrm{C}=$ concentración de proteína en $\mathrm{mg} / \mathrm{mL}$, $\left.\mathrm{R}^{2}=0.998\right)$.

\section{Análisis de datos}

Una vez probados los supuestos de normalidad y homocedasticidad, se realizaron análisis de varianza de una ruta y la comparación de medias para determinar los valores significativamente diferentes entre los valores máximos de la actividad lacasa y $\mathrm{MnP}$ por cada cepa, medio de cultivo y residuo agroindustrial empleado con el paquete Statgraphics Plus 5.1.

\section{RESULTADOS}

\section{Colecta, aislamiento y actividad enzimática cua- litativa}

A partir de 105 ejemplares colectados, se obtuvieron 70 aislamientos (datos no mostrados) de macromicetos lignícolas nativos del estado de Veracruz, 41 de ellos presentaron actividad lacasa (Fig. 2). Las cepas fueron ubicadas en los géneros Pleurotus, Pycnoporus, Trametes, Stereum, Trichaptum, Ganoderma, Schizophyllum, Lenzites, Xilaria, Daedalea, Cymatoderma, Dictyopanus, Polyporus, Lentinellus, Hexagonia, Phellinus y Pogonomyces. El hongo Cymatoderma elegans SBN4 presentó el mayor IP con un valor de 33.4, seguido por Pleurotus sp. SBN8, Pycnoporus sanguineus ACT1, Pleurotus djamor COA1, P. sanguineus SBN3, Trametes maxima SM9 y Trametes sp. SBN2, con 19.6, 16.0,
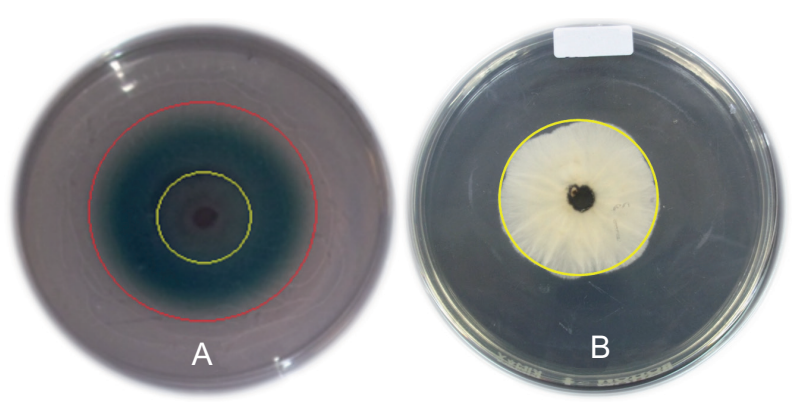

Fig. 2. Valoración cualitativa de la actividad lacasa. A) Cepa con actividad lacasa y B) Cepa sin actividad lacasa. El círculo rojo corresponde al halo de oxidación del ABTS y el círculo amarillo corresponde al crecimiento diametral del micelio del hongo 
15.2, 13.8, 13.0 y 12.9, respectivamente (Fig. 3). Sin embargo, a pesar de que C. elegans SBN4 presentó el IP más alto, no fue seleccionado para los estudios subsecuentes debido a que produjo una escasa actividad lacasa en la evaluación cuantitativa (datos no mostrados). Por lo tanto, únicamente fueron seleccionados los aislamientos Pycnoporus sanguineus ACT1 (IP = 16.0), Trametes maxima SM9 (IP = 13.0) y Daedalea elegans PM7 (IP = 4.9), los cuales presentaron una suficiente y confiable actividad lacasa en una prueba cuantitativa. La determinación del IP empleando el halo de coloración del ABTS y el diámetro de la colonia resultó una herramienta fácil, rápida y económica para seleccionar los aislamientos que poseen la actividad lacasa. El valor del IP es un resultado arbitrario y no determina el potencial de un aislamiento para la producción de lacasa en fermentación sólida o líquida.

\section{Actividad enzimática ligninolítica en fermenta- ción líquida}

En este ensayo la cepa T. maxima SM9 mostró la mayor actividad lacasa a los $7 \mathrm{~d}$ en el medio de cultivo Sivakumar con $9121.8 \mathrm{U} / \mathrm{mg}$ de proteína (32867.9 U/L,
Fig. 4E). Este valor fue significativamente mayor $(F=25.3, P=0.001)$ a las máximas actividades encontradas en el mismo medio de cultivo con $D$. elegans PM7 (Fig. 4A) y P. sanguineus ACT1 (Fig. 4C), al presentar una actividad lacasa de $1784.2 \mathrm{U} / \mathrm{mg}$ proteína (4349.4 U/L, a los 7 d) y $5422.2 \mathrm{U} / \mathrm{mg}$ proteína (16 256.5 U/L, a los $8 \mathrm{~d}$ ), respectivamente.

En cuanto a la actividad MnP ocurrió algo similar a la actividad lacasa. En el medio de cultivo Sivakumar con la cepa T. maxima SM9 se obtuvo la mayor actividad de la enzima con $477.9 \mathrm{U} / \mathrm{mg}$ de proteína (1700.8 U/L) a los $7 \mathrm{~d}$ de cultivo (Fig. 4F). Este valor fue significativamente mayor $(F=37.2, P=0.0004)$ a las máximas actividades obtenidas en el mismo medio de cultivo con D. elegans PM7 (Fig. 4B) y $P$. sanguineus ACT1 (Fig. 4D), al mostrar una actividad MnP de $40.0 \mathrm{U} / \mathrm{mg}$ de proteína (83.8 U/L, a los 8 d) y $41.0 \mathrm{U} / \mathrm{mg}$ de proteína (146.0 U/L, a los $5 \mathrm{~d})$, respectivamente.

En los medios de cultivo Bose y Koroljova se detectaron bajas actividades lacasa y MnP para los tres aislamientos evaluados. En el medio de cultivo Bose la mayor actividad lacasa fue de $6.7(22.0 \mathrm{U} / \mathrm{L}$, a $\operatorname{los} 8 \mathrm{~d}), 3.1$ (7.9 U/L, a los 3 d) y 12.9 (28.6 U/L, a

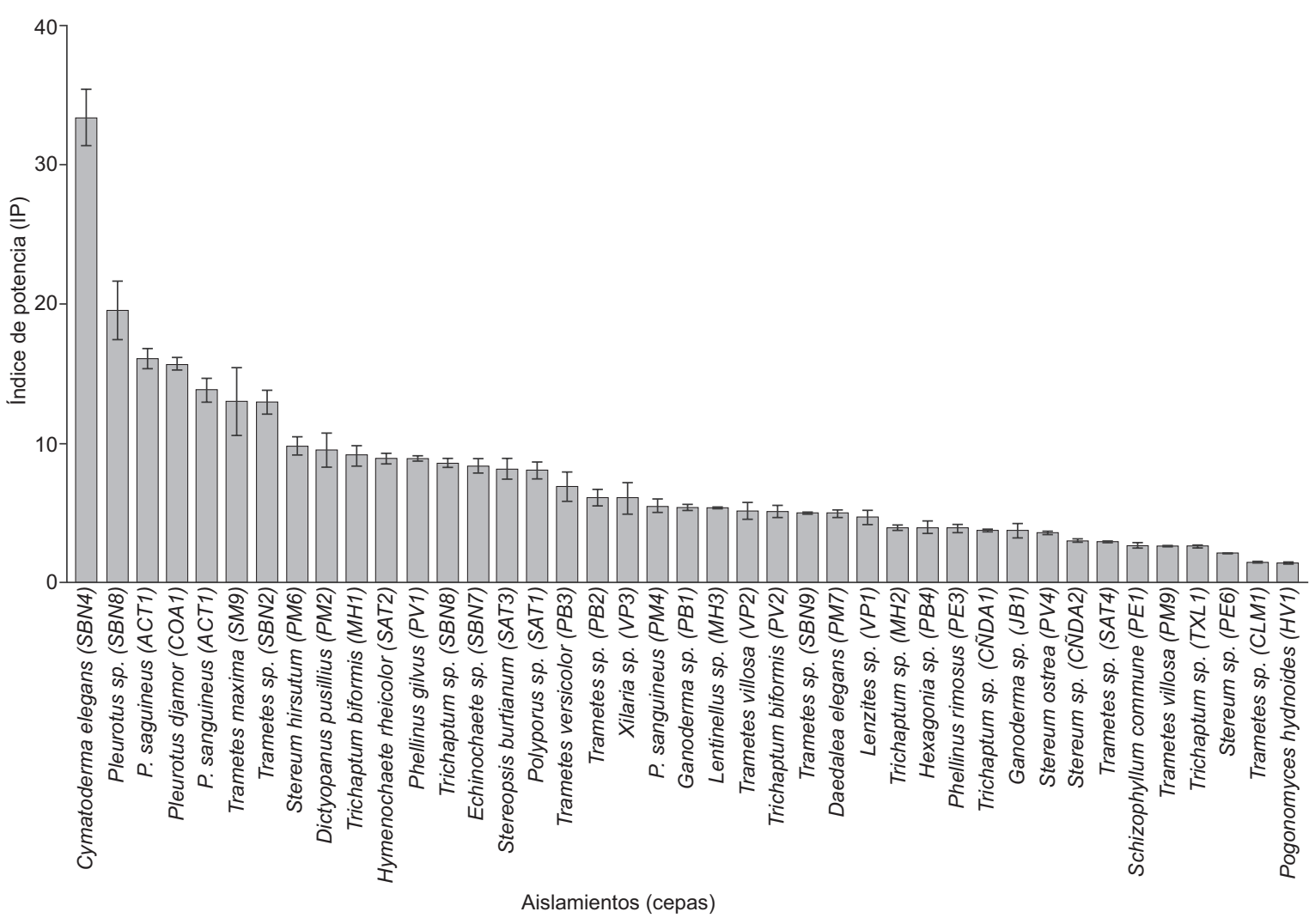

Fig. 3. Índice de potencia de la actividad cualitativa de lacasa en macromicetos lignícolas nativos del estado de Veracruz, México (media \pm error estándar, $n=4)$. 

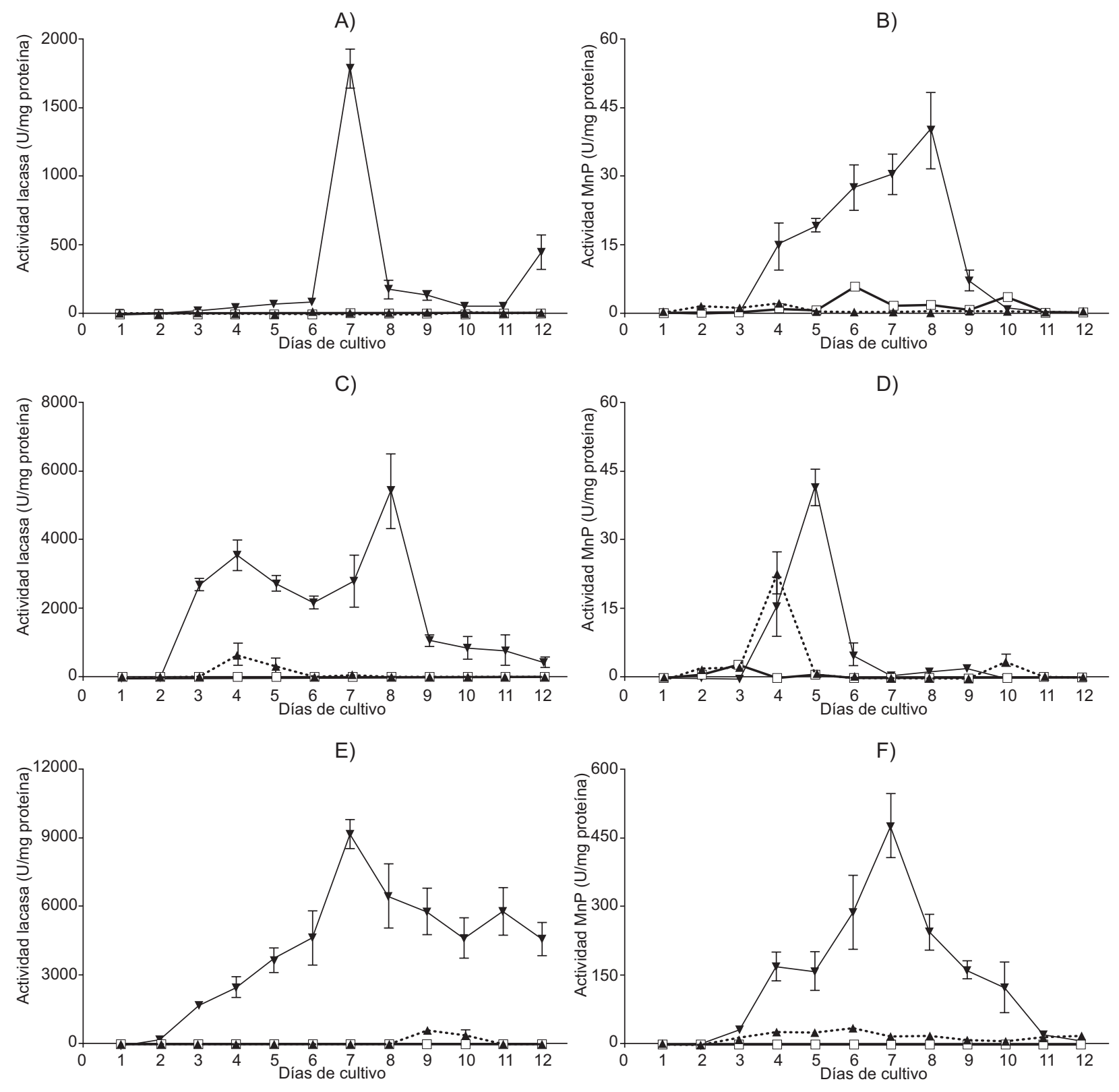

$\rightarrow-$ Bose $\cdot$ Koroljova $\rightarrow$ Sivakumar

Fig. 4. Actividad lacasa y manganeso peroxidasa (MnP) de Daedalea elegans PM7 (A y B), Pycnoporus sanguineus ACT1 (C y D) y Trametes maxima SM9 (E y F) en tres medios de cultivo en fermentación líquida: Bose ( $\square$ ), Koroljova ( $)$ y Sivakumar $(\Delta)$. Media \pm error estándar $(\mathrm{n}=3)$.

los 12 d) U/mg de proteína para D. elegans PM7, $P$. sanguineus ACT1 y T. maxima SM9, respectivamente (Fig. 4A, C y E). Mientras que para la actividad MnP, la mayor fue de $5.5(16.2 \mathrm{U} / \mathrm{L}$, a $\operatorname{los} 6 \mathrm{~d}), 2.7$ (7.0 $\mathrm{U} / \mathrm{L}$, a los $3 \mathrm{~d})$ y $1.3(3.3 \mathrm{U} / \mathrm{L}$, a los $8 \mathrm{~d}) \mathrm{U} / \mathrm{mg}$ de proteína para D. elegans PM7, P. sanguineus ACT1 y T. maxima SM9, respectivamente (Fig. 4B, D y F). En cuanto a la actividad lacasa en el medio de cultivo Koroljova, las máximas fueron de 13.6 (29.4 U/L, a $\operatorname{los} 10 \mathrm{~d}), 673.0$ (1379.9 U/L, a los 4 d) y 604.5 (1899.7 U/L, a los 9 d) U/mg de proteína en $D$. elegans PM7, P. sanguineus ACT1 y T. maxima SM9, respectivamente (Fig. 4A, C y E). Por su parte, las máximas actividades de la MnP en el medio Koroljova para $D$. elegans PM7, P. sanguineus ACT1 y $T$. maxima SM9 fueron de 1.8 (3.6 U/L, a los $4 \mathrm{~d}$ ), 22.8 (43.7 U/L, a los 4 d) y $37.6(73.0 \mathrm{U} / \mathrm{L}$, a $\operatorname{los} 6 \mathrm{~d}) \mathrm{U} /$ mg de proteína, respectivamente (Fig. 4B, D y F). 


\section{Actividad enzimática ligninolítica en fermenta- ción sólida}

Se evaluó la actividad lacasa y MnP de los aislamientos $D$. elegans PM7, P. sanguineus ACT1 y $T$. maxima SM9 sobre paja de trigo, bagazo de caña y aserrín de pino. En este ensayo la máxima actividad lacasa se obtuvo con el hongo $P$. sanguineus ACT1 cultivado sobre paja de trigo (a los $15 \mathrm{~d}$ ) y sobre bagazo de caña (a los $6 \mathrm{~d}$ ) con $1409.0 \mathrm{U} / \mathrm{mg}$ de peso seco
(118.2 U/L) y $1404.8 \mathrm{U} / \mathrm{mg}$ de peso seco (76.7 U/L), respectivamente (Fig. 5C). Ambos valores fueron estadísticamente mayores $(F=12.9, P=0.002)$ a la máxima actividad lacasa de D. elegans PM7 (a los $21 \mathrm{~d}$ ) obtenida sobre paja de trigo con $133.9 \mathrm{U} / \mathrm{mg}$ de peso seco (27.2 U/L; Fig. 5A). Sin embargo, la máxima actividad lacasa de $T$. maxima SM9 obtenida en bagazo de caña $(874.4 \mathrm{U} / \mathrm{mg}$ de peso seco $=62.1 \mathrm{U} / \mathrm{L})$ a los 3 d de cultivo (Fig. 5E) fue estadísticamente
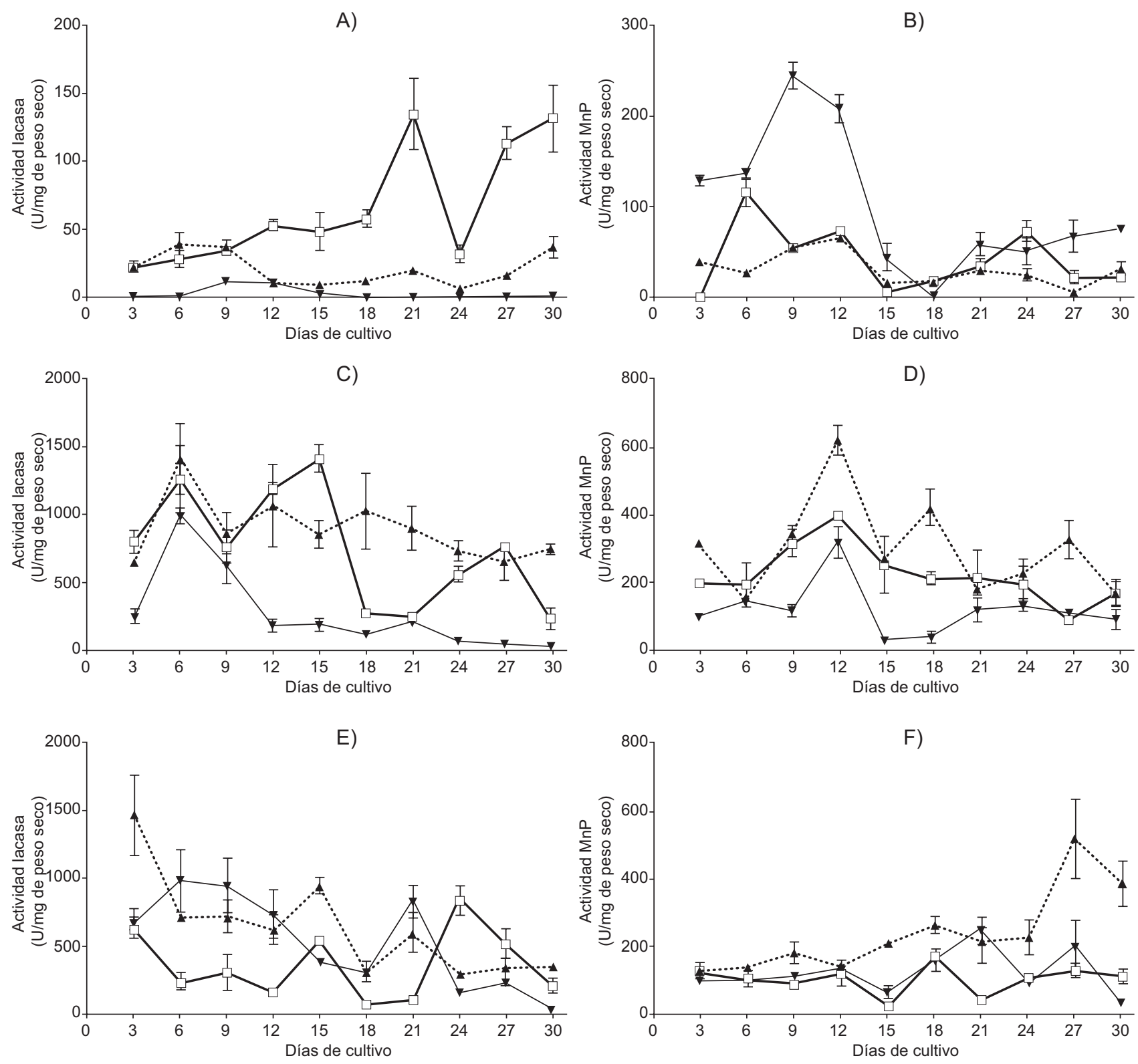

$-\square$ Paja de trigo $\cdots$ Bagazo de caña $\rightarrow$ Aserrín de pino

Fig. 5. Actividad lacasa y manganeso peroxidasa (MnP) de Daedalea elegans PM7 (A y B), Pycnoporus sanguineus ACT1 (C y D) y Trametes maxima SM9 (E y F) en fermentación sólida en tres residuos agroindustriales: paja de trigo ( $\square$ ), bagazo de caña ( $\nabla)$ y aserrín de pino $(\Delta)$. Media \pm error estándar $(n=3)$. 
igual $(F=2.54, P=0.159)$ a las máximas actividades obtenidas por $P$. sanguineus ACT1 cultivado sobre paja de trigo $(1409.0 \mathrm{U} / \mathrm{mg}$ de peso $\mathrm{seco}=118.2 \mathrm{U} / \mathrm{L})$ y bagazo de caña $(1404.8 \mathrm{U} / \mathrm{mg}$ de peso seco $=76.7 \mathrm{U} / \mathrm{L}$; Fig. 5C). Aún sobre aserrín de pino, $P$. sanguineus ACT1 $(990.4 \mathrm{U} / \mathrm{mg}$ de peso seco $=100.1 \mathrm{U} / \mathrm{L}$, a los 6 d, Fig. 5C) obtuvo significativamente $(F=32.9$, $P=0.006)$ una mayor actividad lacasa en comparación con T. maxima SM9 (587.9 U/mg de peso seco $=60.2$ U/L, a los 6 d, Fig. 5E) y D. elegans PM7 $(11.8 \mathrm{U} / \mathrm{mg}$ de peso seco $=2.1 \mathrm{U} / \mathrm{L}$, a los $9 \mathrm{~d})$ establecidos sobre el mismo sustrato (Fig. 5A).

En cuanto a la actividad MnP, el bagazo de caña resultó mejor sustrato para la producción de la enzima para los hongos $P$. sanguineus ACT1 (a los $12 \mathrm{~d}$ ) y T. maxima SM9 (a los 27 d) con $619.0(33.8 \mathrm{U} / \mathrm{L})$ y $519.6(36.9$ U/L) U/mg de peso seco (Fig. 5D y F), respectivamente. Ambos valores fueron significativamente mayores $(F=16.3, P=0.003)$ a la máxima actividad MnP de $D$. elegans PM7 (Fig. 5B) producida en el mismo sustrato $(65.6 \mathrm{U} / \mathrm{mg}$ de peso seco $=18.2 \mathrm{U} / \mathrm{L})$ a los 12 d. En cuanto a la paja de trigo, resultó ser significativamente $(F=76.26, P=0.0001)$ un mejor sustrato para la producción de $\mathrm{MnP}$ en $P$. sanguineus ACT1 (397.0 U/mg de peso seco $=33.3 \mathrm{U} / \mathrm{L}$, a los 12 d, Fig. 5D), aunque no con T. maxima SM9 $(175.8 \mathrm{U} / \mathrm{mg}$ de peso $\mathrm{seco}=30.6 \mathrm{U} / \mathrm{L}$, a los $18 \mathrm{~d}$, Fig. 5F $)$ y D. elegans PM7 (115.4 U/mg de peso seco $=23.4 \mathrm{U} / \mathrm{L}$, a los 6 d, Fig. 4B). Mientras que para el aserrín de pino, al comparar las máximas actividades, no se encontró diferencia significativa $(F=1.27, P=$ $0.34)$ para la producción de $\mathrm{MnP}$ en los tres hongos evaluados, las actividades $\mathrm{MnP}$ fueron de 243.5 (13.6 U/L), $317.4(32.0 \mathrm{U} / \mathrm{L})$ y $250.8(25.6 \mathrm{U} / \mathrm{L})$ U/mg de peso seco para D. elegans PM7 (a los 9 d, Fig. 5B), P. sanguineus ACT1 (a los 12 d, Fig. 5D) y T. maxima SM9 (a los 21 d, Fig. 5F), respectivamente.

\section{DISCUSIÓN}

En el presente estudio se cuantificó la actividad enzimática ligninolítica de macromicetos nativos del estado de Veracruz. Hasta la fecha son limitados los estudios enfocados en la búsqueda de especies fúngicas con alto potencial para la producción de enzimas ligninolíticas (Tapia-Tussell et al. 2011). Una herramienta fácil y económica para conocer la habilidad de una cepa para producir enzimas ligninolíticas como la lacasa, es la determinación cualitativa a través del halo de oxidación del ABTS (Rubilar-Araneda 2007, Sunil et al. 2011). Con este halo se calculó el índice de potencia (IP), definido como la relación del halo de oxidación y el crecimiento del micelio, este IP ha sido empleado en estudios similares (MárquezOrtega 2004, Cruz-Ramírez et al. 2012, AntonioRevuelta 2013). Sin embargo, el IP es un valor que sobreestima la actividad enzimática de una cepa, al no dar un valor preciso de su actividad. En este trabajo se observó que la cepa con mayor IP (Cymatoderma elegans SBN4) no fue precisamente la que produjo más actividad lacasa en fermentación líquida o sólida, de hecho no fue seleccionada para tales estudios al presentar la mínima actividad lacasa en el cultivo líquido. La oxidación del ABTS y la formación del halo de oxidación, puede haber sido ocasionada por otras peroxidasas fúngicas (aril-alcohol oxidasa, glucosa oxidasa) o por radicales libres $\left(\mathrm{H}_{2} \mathrm{O}_{2} \mathrm{u} \mathrm{OH} \mathrm{OH}^{-}\right)$.

$\mathrm{La}$ actividad lacasa y $\mathrm{MnP}$ en fermentación líquida se vio favorecida en el medio de cultivo Sivakumar en las tres especies evaluadas. El medio de cultivo Sivakumar posee la misma fuente y cantidad de carbono (glucosa) que el medio Bose. Sin embargo, posee menos cantidad de nitrógeno $\left(\left(\mathrm{NH}_{4}\right)_{2} \mathrm{SO}_{4} \mathrm{y}\right.$ extracto de levadura), $\mathrm{CaCl}_{2}, \mathrm{MgSO}_{4}$ y $\mathrm{KH}_{2} \mathrm{PO}_{4}$. Es posible que la baja concentración de estos elementos en el medio de cultivo Sivakumar favoreciera la producción de ambas enzimas. Las enzimas ligninolíticas son producidas durante el metabolismo secundario bajo condiciones limitadas de nitrógeno (Kaal et al. 1995). Asimismo, en macromicetos lignícolas Jegatheesan et al. (2012) y Chan-Cupul et al. (2014) han reportado que concentraciones bajas de $\mathrm{CaCl}_{2}$ favorecen la actividad lacasa $(0.1 \mathrm{mg} / \mathrm{L})$ y $\mathrm{MnP}(0.01 \mathrm{mg} / \mathrm{L})$, respectivamente. La función del $\mathrm{CaCl}_{2}$ en el medio de cultivo es mantener la estructura y estabilidad de ambas enzimas. Del mismo modo, Stoilova et al. (2010) sugieren que concentraciones bajas de $\mathrm{MgSO}_{4}$ contribuyen a mantener la estabilidad de la lacasa durante periodos prolongados de incubación. Sin embargo, las altas concentraciones de sales $\left(\left(\mathrm{NH}_{4}\right)_{2} \mathrm{SO}_{4}, \mathrm{CaCl}_{2}, \mathrm{MgSO}_{4}\right.$ y $\left.\mathrm{KH}_{2} \mathrm{PO}_{4}\right)$ en el medio de cultivo incrementan su potencial osmótico y en consecuencia, se puede reducir el crecimiento del micelio (Beever y Laracy 1986).

Aunado a lo anterior, el medio de cultivo Sivakumar, a diferencia del medio Bose, posee $\mathrm{CuSO}_{4}(0.001 \mathrm{~g} / \mathrm{L})$ $\mathrm{y} \mathrm{MnSO}_{4}(0.002 \mathrm{~g} / \mathrm{L})$ en bajas concentraciones, ambos elementos son conocidos como inductores de lacasa (Fonseca et al. 2010) y MnP (Kamitsuji et al. 2005), respectivamente. El medio de cultivo Koroljova posee $50 \%$ menos contenido de carbono (glucosa) en comparación con los medios Bose y Sivakumar. El contenido de carbono y el tipo de fuente son factores que influyen en la producción de enzimas ligninolíticas (Elisashvili et al. 2011). Por lo tanto, podría ser que el 
bajo contenido de carbono en el medio Koroljova sea la razón de haber encontrado menos actividad lacasa y $\mathrm{MnP}$ en comparación con el medio Sivakumar. Además, el medio Koroljova carece de $\mathrm{CuSO}_{4}$, compuesto inductor de lacasas (Shutova et al. 2008).

Los valores obtenidos indican que la cepa de $T$. maxima SM9 presenta una alta producción de lacasa, al menos en el medio de cultivo Sivakumar. La maxima actividad lacasa alcanzada por T. maxima SM9 se obtuvo a los $7 \mathrm{~d}$ de cultivo $(9121.8 \mathrm{U} / \mathrm{mg}$ de proteína $=32867.9 \mathrm{U} / \mathrm{L}$ ), valor mucho mayor que otras reportadas para la misma especie por Songulashvili et al. (2007) y Elisashvili et al. (2011) con 13500.0 y $8600.0 \mathrm{U} / \mathrm{L}$, respectivamente. Es bien conocido que el género Trametes es productor de lacasas, entre las especies reportadas están Trametes versicolor (1200 U/L, Rodríguez-Couto et al. 2002), Trametes ochracea (3900.0 U/L, Elisashvili et al. 2011) y Trametes trogii (14200.0 U/L, Zouari et al. 2006). Sin embargo, los valores reportados son menores a los obtenidos con T. maxima SM9. Asimismo, $T$. maxima SM9 resultó excelente productor de $\mathrm{MnP}$ en el medio de cultivo Sivakumar con $477.9 \mathrm{U} / \mathrm{mg}$ proteína $(=1700.8 \mathrm{U} / \mathrm{L})$, valor superior al reportado para una cepa de T. maxima $(610 \mathrm{U} / \mathrm{L})$ por Elisashvili et al. (2011). Inclusive, otras especies de Trametes presentan menor actividad MnP, por ejemplo: Trametes zonata (360 U/L, Songulashvili et al. 2007), Trametes unicolor (590 U/L, Elisashvili et al. 2011) y Trametes versicolor (150 U/L, Lorenzo et al. 2002).

Adicionalmente, P. sanguineus ACT1 destacó por su buena producción de lacasa y MnP. Al igual que con T. maxima SM9, el medio de cultivo Sivakumar permitió la mayor producción de dicha enzima por P. sanguineus ACT1. Las máximas actividades para lacasa y MnP fueron de $5422.2 \mathrm{U} / \mathrm{mg}$ de proteína (16256.5 U/L) y $41.5 \mathrm{U} / \mathrm{mg}$ de proteína (146.0 U/L), respectivamente. La actividad lacasa obtenida por $P$. sanguineus ACT1 es ligeramente menor a la actividad reportada por Ramírez-Cavazos et al. (2014) con una cepa nativa del noreste de México, la cual presentó $20000 \mathrm{U} / \mathrm{L}$ en un medio enriquecido con jugo de tomate y glucosa/bactopeptona. La actividad MnP de $P$. sanguineus ha sido menos estudiada en comparación con la de la lacasa. Al respecto, Watanabe et al. (2012) reportaron una baja actividad de $\mathrm{MnP}(0.08$ ABS/min) con una cepa de $P$. sanguineus cultivada en efluentes de la industria farmacéutica. Sin embargo, los valores reportados por Watanabe et al. (2012) no se pueden comparar con los de este estudio debido a que fueron presentados con una unidad de medición (ABS/min) diferente a la empleada en este trabajo (U/L y U/mg de proteína).
La actividad enzimática ligninolítica (lacasa y $\mathrm{MnP}$ ) en macromicetos lignícolas depende de la composición del medio de cultivo empleado para su producción y es regulada por diversos nutrientes tales como la glucosa, nitrógeno y iones metálicos $\left(\mathrm{Cu}^{2+}\right.$, $\left.\mathrm{Mn}^{2+}, \mathrm{Mg}^{2+}, \mathrm{Fe}^{2+}\right)$ (Elisashvili et al. 2011). Además, la producción de lacasa y $\mathrm{MnP}$ se ve afectada por numerosos factores involucrados en el cultivo tales como la composición y la fuente de nutrientes $(\mathrm{C} \mathrm{y} \mathrm{N})$, el $\mathrm{pH}$, la temperatura, la agitación y la presencia de inductores como etanol, alcohol veratrílico, compuestos análogos de la lignina, etcétera (Rodríguez-Couto et al. 2002).

La fermentación sólida para la producción de enzimas resulta más económica que la fermentación líquida en medios de cultivo con ausencia de inductores enzimáticos (Demir et al. 2011). Por lo tanto, si se desea producir enzimas con menor impacto ambiental y a menor costo, la fermentación sólida es una alternativa a considerar. Los resultados del presente estudio indican que la paja de trigo permitió la mayor actividad lacasa en D. elegans PM7 (133.8 U/mg de peso seco a los $21 \mathrm{~d}$ ) y $P$. sanguineus ACT1 (1408.9 U/mg de proteína a los $15 \mathrm{~d})$, mientras que en el bagazo de caña, T. maxima SM9 produjo su máxima actividad lacasa $(874.4 \mathrm{U} / \mathrm{mg}$ de peso seco a los 3 d). En cuanto a la actividad MnP, las cepas $P$. sanguineus ACT1 (619.0 U/mg de peso seco a los $12 \mathrm{~d})$ y $T$. maxima SM9 (519.5 U/mg de peso seco a los $27 \mathrm{~d}$ ), presentaron la mayor actividad MnP sobre bagazo de caña. Para D. elegans PM7, en el aserrín de pino fue que se produjo la mayor actividad $\mathrm{MnP}$ (243.4 U/mg de peso seco a los 9 d). En fermentación sólida $P$. sanguineus ACT1 fue el hongo con mayor actividad lacasa y $\mathrm{MnP}$, dejando en segundo término a T. maxima SM9 y D. elegans PM7. En la fermentación líquida fue T. maxima SM9 el hongo con mayor actividad lacasa, dejando en segundo término a $P$. sanguineus ACT1 y D. elegans PM7.

La composición química de los sustratos agroindustriales difiere entre sí. De acuerdo con el trabajo de López-Miranda et al. (2009) y Prinsen (2010), la paja de trigo (16.85\%) y el bagazo de caña $(23.09 \%)$ poseen menos lignina en comparación con el aserrín de pino $(26.5 \%)$, y puede ser que el menor contenido de lignina en la paja de trigo y el bagazo de caña facilite la accesibilidad a la celulosa, fuente de carbono para el crecimiento fúngico y síntesis enzimática. Esto da como resultado que la paja de trigo sea el mejor sustrato para la producción de lacasa, al menos en $D$. elegans PM7 y P. sanguineus ACT1. Por otra parte, el bagazo de caña resultó mejor sustrato para la producción de MnP, al menos en P. sanguineus ACT1 y en 
T. maxima SM9. Mientras que D. elegans PM7 prefirió el aserrín de pino. Sin embargo, no se debe descartar que la síntesis de enzimas ligninolíticas es una característica especies-cepa dependiente y que, además, la fuentes y composiciones nutrimentales tanto de cultivos líquidos y sustratos agroindustriales son un factor más a considerar en la producción de enzimas.

Este trabajo es una plataforma para continuar estudiando la micobiota nativa del estado de Veracruz, si se desea conservar el germoplasma fúngico a través de ceparios ante la constante pérdida de diversidad. Además de ser una antesala necesaria para los estudios sobre micorremediación en ambientes acuáticos y edáficos contaminados en el estado de Veracruz.

\section{CONCLUSIONES}

Por la amplia diversidad fúngica que prolifera en el estado en Veracruz es factible encontrar ejemplares de basidiomicetos lignícolas con potencial para la producción de enzimas ligninolíticas. Cuando se tiene un gran número de cepas fúngicas, la determinación del IP empleando la detección cualitativa de lacasas a través del halo de oxidación del ABTS, es una herramienta fácil, rápida y económica para seleccionar cepas productoras de lacasa. Sin embargo, es necesario realizar una evaluación cuantitativa en fermentación líquida para determinar si la cepa realmente produce la enzima. En fermentación líquida la cepa T. maxima SM9 destacó por su alta producción de lacasa y MnP. En el medio de cultivo Sivakumar las especies probadas tuvieron la mayor producción de lacasa y MnP. En fermentación sólida, $P$. sanguineus ACT1 destacó por su alta actividad lacasa tanto en paja de trigo como en bagazo de caña. Mientras que para la actividad MnP, las especies indicadas para producir la enzima son T. maxima SM9 y $P$. sanguineus ACT1 y el sustrato ideal para ello es el bagazo de caña.

\section{REFERENCIAS}

Aguirre-Acosta E., Ulloa M., Aguilar S., Cifuentes J. y Valenzuela R. (2014). Biodiversidad de hongos en México. Rev. Mex. Biodivers. Supl. 85, 76-81. DOI: $10.7550 / \mathrm{rmb} .33649$.

Antonio-Revuelta B. (2013). Evaluación del potencial enzimático de macromicetos lignícolas del centro del estado de Veracruz. Tesis de Licenciatura, Facultad de Ciencias Biológicas y Agropecuarias. Universidad Veracruzana. Xalapa, Veracruz, México, 38 pp.
Beever R.E. y Laracy E.P. (1986). Osmotic adjustment in the filamentous fungus Aspergillus nidulans. J. Bacteriol. 168, 1358-1365.

Bose S., Mazumder S. y Mukherjee M. (2007). Laccase production by the white-rot fungus Termitomyces clypeatus. J. Basic Microbiol. 47, 127-131.

DOI: 10.1002/jobm.200610206.

Chan-Cupul W. (2014). Bioprospección enzimática de hongos nativos en co-cultivo y su valoración en la biorremediación de un suelo contaminado con atrazina. Tesis de Doctorado. Instituto de Ecología A. C. (INECOL). Xalapa, Veracruz, México, 205 pp.

Chan-Cupul W., Heredia-Abarca G., Martínez-Carrera D. y Rodríguez-Vázquez R. (2014) Enhancement of ligninolytic enzyme activities in a Trametes maximaPaecilomyces carneus co-culture: key factors revealed after screening using a Plackett-Burman experimental design. Electron. J. Biotechnol. 17, 114-121.

DOI: 10.1016/j.ejbt.2014.04.007.

Chaparro D.F., Rosas D.C. y Varela A. (2009). Aislamiento y evaluación de la actividad enzimática de hongos descomponedores de madera (Quindío, Colombia). Rev. Iberoam. Micol. 26, 238-246. DOI: 10.1016/j.riam.2009.03.005.

Cruz-Ramírez M.G., Rivera-Ríos J.M., Téllez-Jurado A., Maqueda-Gálvez A.P., Mercado-Flores Y. y AranaCuenca A. (2012). Screening for thermotolerant ligninolytic fungi with laccase, lipase, and protease activity in Mexico. J. Environ. Manage. 95, 256-259. DOI: 10.1016/j.jenvman.2010.10.045.

Demir A., Aytar P., Gedikli S., Cabuk A. y Arisoy M. (2011). Laccase production with submerged and solid state fermentation: benefit and cost analysis. Hacettepe J. Biol. Chem. 39, 305-313.

Elisashvili V., Torokb T., Kachlishvilia E., Khardziania T., Metrevelo E., Kobakhidze A. y Berikashvili I. (2011). Evaluation and regulation of lignocellulolytic activity of novel white-rot basidiomycetes. Glob. J. Biotechnol. Biochem. 2, 134-141.

Fernández-Fernández M., Sanromán M.A. y Moldes D. (2013). Recent developments and applications of immobilized laccase. Biotech. Adv. 31, 1808-1825. DOI: 10.1016/j.biotechadv.2012.02.013.

Fonseca M.I., Shimizu E., Zapata P.D. y Villalba L.L. (2010). Copper inducing effect on laccase production of white rot fungi native from Misiones (Argentina). Enzyme Microb. Technol. 46, 534-539.

DOI: 10.1016/j.enzmictec.2009.12.017.

Gilbertson R.L. y Ryvarden L. (1987). North American polypores. 2a ed. Megasporoporia-Wrightoporia, FungiFlora. Oslo, Noruega, 885 pp. DOI: 10.2307/3759470.

Glenn J.K. y Gold M.H. (1983). Decolorization of several polymeric dyes by the lignin-degrading basidiomycetes 
Phanerochaete chrysosporium. Appl. Environ. Microbiol. 45, 1741-1747.

Guzmán G. (1995). La diversidad de hongos en México. Revista Ciencias 39, 52-57.

Guzmán G. (1998). Inventorying the fungi of Mexico. Biodivers. Conserv. 7, 369-384.

DOI: $10.1023 / \mathrm{A}: 1008833829473$.

Guzmán G. (2008). Hongos de parques y jardines y su relación con la gente. 1a ed. Secretaría de Educación del Estado de Veracruz. Xalapa, Veracruz, México. 243 pp.

Harris-Valle C., Valenzuela-Soto E., Sánchez A., GaitánHernández R. y Esqueda M. (2014). Variability in the ligninolytic enzymes activity by Lentinula edodes in submerged culture with lignin and glucose. American Journal of Agricultural and Biological Science 9, 369378. DOI: 10.3844/ajabssp.2014.369.378.

Hernández-Luna C.E., Gutiérrez-Soto G. y SalcedoMartínez S.M. (2007). Screening for decolorization basidiomycetes in Mexico: Screening and selection of ligninolytic basidiomycetes with ability in Northeast Mexico. World J. Microbiol. Biotechnol. 24, 465-473. DOI: 10.1007/s11274-007-9495-3.

Huifang X., Li Q., Wang M. y Zhao L. (2013). Production of a recombinant laccase from Pichia pastoris and biodegradation of chlorpyrifos in a laccase/vanillin system. J. Microbiol. Biotechnol. 23, 864-871.

DOI: $10.4014 / \mathrm{jmb} .1212 .12057$.

Jegatheesan M., Senthilkumaran M. y Eyini M. (2012). Enhanced production of laccase enzyme by the whiterot mushroom fungus Pleurotus florida using response surface methodology. Int. J. Curr. Res. 4, 25-31.

Kaal E.E.J., Field J.A., y Joyce T. W. (1995). Increase ligninolytic enzyme activities in several white-rot basidiomycetes by nitrogen sufficient media. Bioresour. Technol. 53, 133-139.

DOI: 10.1016/0960-8524(95)00066-N.

Kamitsuji H., Honda Y., Watanabe T. y Kuwahara M. (2005). $\mathrm{Mn}^{2+}$ is dispensable for the production of active MnP2 by Pleurotus ostreatus. Biochem. Biophys. Res. Commun. 327, 871-876.

DOI: 10.1016/j.bbrc.2004.12.084.

Koroljova S.V.O., Stepanova E.V., Gavrilova V.P., Morozova O.V., Lubimova N.V., Dzchafarova A.N., Jaropolov A.I. y Makower A. (1998). Purification and characterization of the constitutive form of laccase from the basidiomycete Coriolus hirsutus and effect of inducers on laccase synthesis. Biotechnol. Appl. Biochem. 28, 47-54. DOI: 10.1111/j.1470-8744.1998.tb00511.x.

López-Miranda J., Soto-Cruz N.O., Rutiaga-Quiñones O.M., Medrano-Roldán H. y Arévalo-Niño K. (2009). Optimización del proceso de obtención de azúcares fermentables a partir de aserrín de pino. Rev. Int. Contam. Ambie. 25, 95-102.
Lorenzo M., Moldes M., Rodríguez-Couto S. y Sanromán A. (2002). Improving laccase production by employing different lignocellulosic waste in submerged cultures of Trametes versicolor. Bioresour. Technol. 82, 109-113. DOI: 10.1016/S0960-8524(01)00176-6.

Marco-Urrea E. y Reddy C.A. (2012). Degradation of chloro-organic pollutants by white rot fungi. En: Microbial degradation of xenobiotics (S.N. Singh, Ed). Springer-Verlag, Berlin, Alemania, pp. 31-66.

DOI: 10.1007/978-3-642-23789-8_2.

Márquez-Ortega A.E. (2004). Determinación de patrones de inducción de lacasas en hongo Trametes sp. EUM1. Tesis de Especialización en Biotecnología. Universidad Autónoma Metropolitana. Unidad Iztapalapa, México D. F., 63 pp.

Mata G., Ortega-Sánchez C. y Pérez-merlo R. (2011). Inóculo suplementado: evaluación de un método para optimizar la producción de inóculo para el cultivo de Pleurotus en pulpa de café. Revista Mexicana de Micología 34, 53-61.

Núñez M. y Ryvarden L. (2001). East Asian polypore 2. polyporacea S. lato. Syn. Fungorum 14, 170-522.

Osma J.F., Toca-Herrera J.L. y Rodríguez-Couto S. (2010). Uses of laccase in the food industry. Enzyme Res. DOI: 10.4061/2010/918761

Prinsen P. (2011). Composición química de diversos materiales lignocelulósicos de interés industrial y análisis estructural de sus ligninas. Tesis de Maestría, Facultad de Química, Universidad de Sevilla, Sevilla, España, 82 pp.

Ramírez-Cavazos L.I., Junghanns C., Nair R., CárdenasChavez, Hernández-Luna C., Agathos S.N. y Parra R. (2014). Enhanced production of thermostable laccases from a native strain of Pycnoporus sanguineus using central composite design. J. Zhejiang Univ. Sci. B. $15,343-352$. DOI: $10.1631 /$ jzus.B1300246.

Rodríguez-Couto S., Gundín M., Lorenzo M. y Sanromán A. (2002). Screening of supports and inducers for laccace production by Trametes versicolor in semi-solid-state conditions. Process Biochem. 38, 249-255.

DOI: $10.1016 / \mathrm{S} 0032-9592(02) 00087-0$.

Rubilar-Araneda O. (2007). Biorremediación de suelos contaminados con pentaclorofenol (PCF) por hongos de pudrición blanca. Tesis de Doctorado. Facultad de Ciencias Agropecuarias y Forestales, Universidad de la Frontera, Temuco, Chile, 133 pp.

Ruiz-Dueñas F.J., Fernández-Fueyo E., Barrasa J.M., Hammel K.E. y Martínez A.T. (2014). What basidiomycete genomes teach us bout wood biodegradation mechanism. Memorias. $13^{\text {th }}$ European Workshop on Lignocellulosic and Pulp. Sevilla, España. 24 al 27 de junio de 2014,111-114 pp. 
Ryvarden L. (1991). Genera of polypores: nomenclature and taxonomy. Synopsis Fungorum 5, 1-363. DOI: $10.2307 / 3760304$.

Shutova V.V., Renin V.V., Yu A. y Myakushina A. (2008). The effect of copper ion on the production of laccase by the fungus Lentinus (Panus) tigrinus. Appl. Biochem. Microbiol. 44, 619-623. DOI: $10.1134 / \mathrm{S} 0003683808060100$.

Sivakumar R., Rajedran R., Balakumar C. y Tamilvendan M. (2010). Isolation, screening and optimization of production medium for thermostable laccase production from Ganoderma sp. Int. J. Eng. Sci. Technol. 2, 7133-7141. DOI: 10.1134/S0003683808060100.

Stoilova I., Krastanov A. y Stanchev V. 2010. Properties of crude laccase from Trametes versicolor produced by solid-substrate fermentation. Adv. Biosci. Biotechnol. 1, 208-2015. DOI: 10.4236/abb.2010.13029.

Sunil S.M., Renuka P.S., Pruthvi K., Swetha M., Malini S. y Veena S.M. (2011). Isolation, purification and characterization of fungal laccase from Pleurotus sp. Enzyme Res. DOI: 10.4061/2011/248735.
Tapia-Tussell R., Pérez-Brito D., Rojas-Herrera R., CortesVelázquez C., Rivera-Muñoz G. y Solis-Pereira S. (2011). New laccase-producing fungi isolates with Biotechnology potential in dye decolorization. Afr. J. Biotechnol. 10, 10134-10142. DOI: 10.5897/AJB11.331.

Watanabe R.A.M., Sales P.T.F., Campos L.C., García T.A., Valadares M.C., Schimidt F. y Santiago M.F. (2012). Evaluation of the use of Pycnoporus sanguineus fungus for phenolics and genotoxicity decay of a pharmaceutical effluent treatment. Ambi-Agua 7, 41-50.

DOI: 10.4136/1980-993X.

Zouari M.H., Mechini T., Dhouib A., Sayadi S., Martínez A.T. y Martínez M.J. (2006). Laccase purification and characterization from Trametes trogii isolated in Tunisia: decolorization of textile dyes by purified enzyme. Enzyme Microb. Technol. 39, 141-148. DOI: 10.1016/j.enzmictec.2005.11.027. 